\title{
Sensing Bluetooth Mobility Data: Potentials and Applications
}

\author{
João Filgueiras ${ }^{1}$, Rosaldo J. F. Rossetti ${ }^{2}$, Zafeiris Kokkinogenis ${ }^{2}$, Michel \\ Ferreira $^{3}$, Cristina Olaverri ${ }^{3}$, Marco Paiva ${ }^{3}$, João Manuel R. S. Tavares ${ }^{4}$, and \\ Joaquim Gabriel ${ }^{4}$ \\ ${ }^{1}$ Instituto de Engenharia de Sistemas e Computadores Investigação e \\ Desenvolvimento (INESC-ID), Lisbon, Portugal \\ jfilgueiras@inesc-id.pt \\ 2 LIACC, DEI, Faculty of Engineering, University of Porto, Porto, Portugal \\ zafeiris.kokkinogenis@gmail.com, rossetti@fe.up.pt \\ 3 Instituto de Telecomunicações, University of Porto, Porto, Portugal \\ \{michel, cristina.olaverri, \}@dcc.fc.up.pt, marcogouveia.paiva@gmail.com \\ 4 DEMec, Faculty of Engineering, University of Porto, Porto, Portugal \\ \{tavares, jgabriel\}@fe.up.pt
}

\begin{abstract}
Information related to mobility dynamics constitutes an important factor to be considered in traffic management to improve the efficiency of existing systems. We present a proof-of-concept deployment of sensors using the Bluetooth technology to detect traffic flow conditions. Besides traditional method consisting of a network of stationary sensors, we present a novel approach that uses sensors deployed in moving vehicles. Both approaches complement the most common methods of traffic sensing while being more cost-effective and easily available. Early experimental results show the variety of information available through both approaches from Origin/Destination matrices to travel times and insights into mobility neighborhoods. These matrices are important to improve traffic management increasing the efficiency of urban mobility networks.
\end{abstract}

Keywords: Urban mobility data; Bluetooth sensing; Traffic monitoring

\section{Introduction}

The extended use of traditional roadside traffic sensors involves high implementation and maintenance costs and a limited coverage. As a consequence, in the last years, the traffic and transportation research community has shown a great interest in the use of wireless communications and satellite technologies. In combination with traditional traffic sensing approaches, these technologies allow the development of traffic monitoring and advanced traffic management systems at a lower cost and greater coverage [11].

The study of human mobility as a whole is important when trying to comprehend traffic phenomena and design solutions to traffic-related issues. It becomes 
necessary to account for pedestrian, as well as vehicle, mobility, and to understand the interaction between them.

In the new horizon of the urban system tissue and in the perspective of urban designers, engineers and practitioners, users are becoming the central interest and not mere commodities to be transported. New type of monitoring techniques and new type of sensors must be used to achieve such innovative solutions. Advancements in information and communication technologies made information and data to be ubiquitous and measurable. Vehicles and portable electronic devices, such as smart phones, come out of the factory with more and more sensors. Different kinds of interfaces and services are being devised for the full exploitation of their capabilities. A large-scale urban mobile cloud of data streams is thus formed capable of offering valuable information for city planners, traffic controllers, public transportation authorities, tourists and citizens. In that sense, data can be used to understand how and where people gather, their destinations and the centers of their daily activities. On the other hand, mobility information can be useful for the users themselves when planning their trips and commutes.

Smart phones, in particular, have already been proposed as mobility sensors, and they are currently used by commercial services, such as Google for traffic estimation. But such sensing systems are mostly proprietary and commonly focus on a specific aspect of transportation systems.

The cost effective solution that is offered by using the Bluetooth wireless communications technology can overcome these difficulties. This solution allows mobility monitoring using autonomous stations that continuously search for nearby Bluetooth devices. These devices that can range from mobile phones to hands-free devices embedded in cars can be tracked over networks of sensors because of their unique hardware address. This solution allows monitoring vehicles as well as pedestrians simultaneously.

This work uses the Bluetooth technology to build and deploy a network of fixed and mobile sensors in order to study and characterize urban mobility conditions in the city of Porto, in Portugal. We present our preliminary results with a fixed network of sensors, as well as results using a novel approach to Bluetooth monitoring by deploying sensors in moving vehicles. Long-term experiments using mobile sensors allow an insight into the concept of mobile vehicular neighbors.

Next section revises related work in the areas of traffic monitoring and management using wireless communication technology. Section 3 presents a description of the methodology used to collect and visualize the collected Bluetooth data. Section 4 reports a preliminary evaluation of the monitoring tool. Finally, Section 5 concludes the paper.

\section{Related work}

The use of wireless networks to estimate patterns of human mobility and social dynamics has been a subject of great interest in studies over the past decade. Results show that humans usually follow simple reproducible patterns that have 
a single spatial distribution [4]. Consequently, this behavior model might be reflected in traffic mobility patterns. Information related to Origin/Destination $(\mathrm{O} / \mathrm{D})$ matrices, travel times and route inference to characterize an urban mobility system is needed to achieve an effective traffic management, control and ow optimization. Sensors based on traditional methods for data acquisition lead to a limited coverage and high implementation and maintenance costs. Since high quality traffic information in real time is crucial to develop Intelligent Transportation Systems (ITS), a greater interest for the use of wireless communications and satellite technologies has been shown in the last years [11].

Some recent works discuss the last developments about Floating Car Data based collection methods and applications, focusing on the potential and bottlenecks of this technology are shown [11]. In [1] the author used GPS-based FCD from taxis to dynamically estimate $\mathrm{O} / \mathrm{D}$ matrices and to infer route analysis. The idea behind this study is to use the fleet of taxis as probes to infer mobility patterns. The authors concluded that the data collected through this method was not conclusive enough since it was based on a small sample. Additionally, the original $\mathrm{O} / \mathrm{D}$ matrix of the studied area was unknown and consequently, a general mobility behavior could not be derived. Additional road traffic conditions by GPS reports via GSM network were studied in [7]. The study concluded that high quality travel time could be produced only with a diffusion of samples above $3 \%$ and $5 \%$ in a mid-sized city. Nevertheless, since FCD is real time data, we think that the combination of FCD methods with traditional methods might increase the quality of data resulting on more accurate mobility parameters estimation. Further investigation focusing on the use of cellular network data for inferring real-time mobility information has been conducted through surveys and data classication identifying the advantages and limitations of this method. Particularly, a discussion on the ways to increase the accuracy of known cellular positioning techniques through a traffic congestion estimation service application and studying the trajectory of several thousand of anonymous mobile phone users are found in [20,21]

A new promising technology has been proposed in the traffic and urban planning community based on the interception of Bluetooth Media Access Control (MAC) addresses for the monitoring of traffic conditions that will open new measurement possibilities $[22,23,18,2,5,16]$. The uniqueness of the detected MAC addresses makes the Bluetooth technology particularly appropriate to monitor the mobility conditions. For example, travel times can be determined through the calculation of the difference in time and space from two measurement sites. Malinovskiy et al. [13] compared MAC address based travel times on a corridor equipped with automatic license plate recognition (ALPR) sensors. Authors conclude that Bluetooth sensor are a satisfactory substitute of the traditional monitoring methods, even though the sample size found is significantly smaller than what can be achieved by ALPR systems, when characterizing the actual traffic conditions.

Moreover, through GPS equipment installed in Bluetooth scanner devices, an accurate measurement of distance between successive Bluetooth data collection 
sites can be accomplished, that leads to valuable Origin-Destination (OD) information. This information is obtained by tracing a Bluetooth transceivers path through a series of Bluetooth units with known locations. Barcel et al. present a method to forecast travel times and time-dependent matrices, in uncongested freeway networks, using Bluetooth traces of detected devices [2].

Bluetooth technology only captures the travel time of a fraction of vehicles in the traffic stream. As a consequence, the authors in [17] collected an extensive amount of data on several highways from Maryland and Delaware calculating the average detection rate to be $2 \%$ to $7 \%$. Haseman [6] studied the possibility of using Bluetooth monitoring methods to design work zone traffic control plans. Authors concluded that the measurement on a weekly basis of work zone travel time could provide quantitative data traffic planners can use to evaluate alternative maintenance of traffic techniques, and identify cost-effective practices. They propose also the use of Bluetooth probe tracking as a potential way of traffic contracting methods while strongly advocating for travel mobility when demands warrant.

Tsubota et al. [19] discusses the data filtering aspect of the Bluetooth data collection underlying the importance of the duration that is defined as the time spent by a Bluetooth device to pass through the detection range of a Bluetooth scanner. Young in [24] discusses the insight of the Bluetooth traffic-monitoring project at University of Maryland. Critical issues of design, development, testing, and operations of Bluetooth scanners as real-time traffic sensors are presented in this report.

ONeill in $[15,9,10]$ illustrates how Bluetooth data can be used to understand pedestrian mobility behavior and what is the interaction with the urban environment. Bullock et al [3] verifies the use of Bluetooth sensing technology in tracking pedestrian mobility in public transportation spaces. Specifically shows the adaptability of the technique to measure the transit time through a security checkpoint in an airport environment. [8] used Bluetooth scanners installed on public bus to estimate passengers' $\mathrm{O} / \mathrm{D}$ matrices as an inexpensive approach. In their work, authors estimated that $12 \%$ of the passengers carry a Bluetooth equipped device and the travel data thus collected present $80 \%$ accuracy of the daily fluctuation of the actual flows. Moreover, they conclude that their approach allows characterizing passenger mobility through polycentric networks.

In [12] demonstrated the use of Bluetooth scanners as mobility sensors is feasible for spatial pattern extraction. The authors have applied the technology at social events to monitor a sample of visitors and extract their route choice. Utilizing the Bluetooth approach Liebig et al. were also able to understand microscopic movement behavior.

This work approaches the Bluetooth sensing technology in a twofold way to characterize urban mobility dynamics. In the next section, we discuss a proof-ofconcept deployment of Bluetooth sensors in a static network as well as in moving vehicles in the city of Porto presenting thus a novel way of sensing urban mobility. 


\section{$3 \quad$ Methodology}

We considered two distinct setups for monitoring urban mobility: a) a network of static Bluetooth sensors deployed on key points of the city, and b) mobile Bluetooth sensors deployed on commuting vehicles. In either case, we implement the workflow depicted in Figure 3 and with three stages:

- Data collection

- Data filtering

- Data analysis and visualization

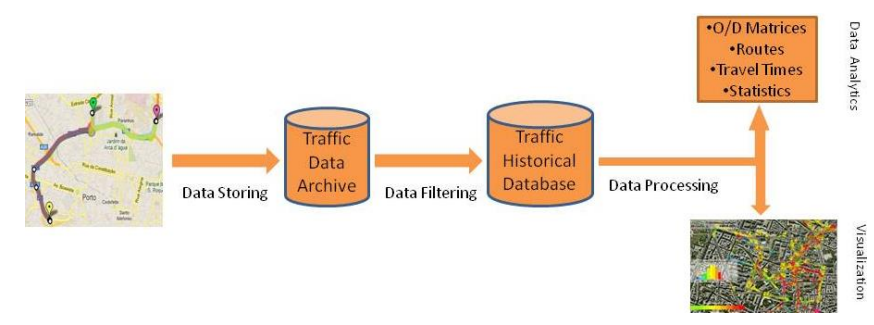

Fig. 1. Data acquisition, filtering and visualization workflow

The sensors, described in the next section, perform data collection and yields lists of devices found in the proximity of the sensors at a given time. The data is then filtered to remove entries that are not of interest, such as static devices from nearby houses that do not relate to mobility. The final sample is stored on a central database where it can be queried to allow data analysis and visualization.

\subsection{Bluetooth Sensors}

Both setups, static and mobile, rely on sensors that implement the Bluetooth protocol to perform an "inquiry" (discovery) to find nearby devices. An inquiry consists of sending a message to all devices within range asking for replies. A device that receives an inquiry message will reply with information about itself: unique hardware address, device type and provided services. The sensors perform discoveries continuously with an interval of 10 seconds as they wait for replies. This behavior can be modified to use just 5 seconds, which might improve the resolution of our data but may also come at the cost of missing some replies. The data collected by each sensor is stored in a pseudo-XML file as depicted in Figure 2. Each discovery has a time stamp, a list of captured devices and, optionally, GPS coordinates.

For each device, we store its unique hardware address and device types, for instance, computer/laptop, or phone/Smartphone. We can also discover the manufacturer of the device by looking at the first half of the hardware address 


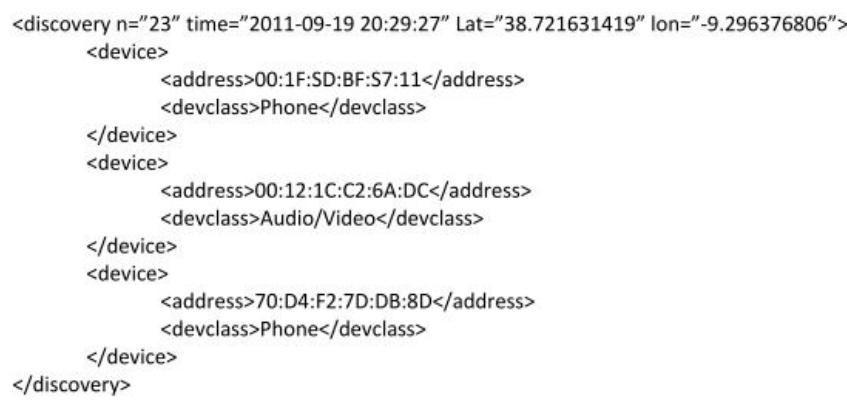

Fig. 2. Output sample of the Bluetooth sensor

that is linked to specific companies. The list of prefixes and correspondent companies can be found at the IEEE website ${ }^{1}$. Having stored discoveries for periods of time, we can then track the movements of any device over space, on each deployed sensor, and over time. Depending on the type of study we want to perform we can filter.

\subsection{Mobility Bluetooth Sensing}

In this setup, a sensor is deployed on a vehicle, continuously capturing device information while the vehicle moves. This type of monitoring will capture devices inside nearby vehicles, carried by pedestrians or, not very often, stationary devices. The resulting data complements what we obtain in a more traditional fashion and gives us a different point of view over urban mobility. Deploying these sensors on a fleet of vehicles could yield traffic volumes for areas where there are no static sensors. However, there are other uses for this data.

An interesting example would be the study of car-pooling (or car-sharing) system feasibility. Car-pooling refers to the idea of sharing a car with a potential stranger during daily commutes with the objective of reducing fuel and other associated to the journey costs. Daily tracks of commutes collected with this setup may show similar routes taken at similar times by different vehicles. Thus, the drivers of these vehicles share a routine and are likely to benefit from car-pooling. Another example would be the study of reputation-based systems on Vehicular Ad-hoc Networks (VANET). In this case, we want to know the number of devices that we recognize in daily routines, therefore, allowing for an actual reputation to be built. Thus, the reading of the mobile sensor could be used as a first way of measuring the association and bond among neighbors building the reputation-based mechanisms. This idea is inspired by the concept of homophily [14], a tendency for members of a network to have a stronger link if they share something in common. In this case, they would share mobility patterns. A stronger link between two members would mean a higher level of trust

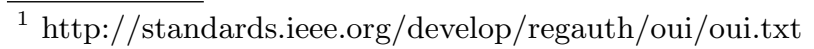


in the information (and eventually services) one shares with the other. Leveraging on this concept, drivers would be able to identify the sources fellow-drivers that will provide him with correct information and services in a VANET formed environment.

\subsection{Static network Bluetooth scanners}

Networks of static sensors constitute the traditional approach when using Bluetooth for traffic monitoring [23]. Here, a number of Bluetooth sensors are installed in key points of a traffic network. This setup allows the collection of data that helps characterizing the traffic network macroscopically.

Effective mobility management, control and flow optimization can benefit from valuable travel information such as Origin/Destination (O/D) matrices, travel times and route inference. Since we can track individual devices, we can build $\mathrm{O} / \mathrm{D}$ matrices and by measuring the time drivers took to reach one sensor after being captured by another, we can estimate travel times. The O/D matrices can be both a) static that is, based simply on device counts, and b) dynamically time-dependent, derived by tracing a Bluetooth device through a series of sensors with known locations.

Considering that a sensor will continuously emit discoveries, we can register the number of consecutive discoveries in which the same device is captured. If a device stays in range for a longer period of time, then it will appear in more consecutive discoveries. Therefore, a higher number of average consecutive discoveries will mean that most devices are spending more time in range, indicating slower local traffic.

\section{Preliminary Results}

This section presents preliminary results using the two aforementioned setups. First measurements show that approximately one vehicle in five contains some type of Bluetooth device that can be detected. The quantity of data from Bluetooth sensors is typically good enough to estimate $\mathrm{O} / \mathrm{D}$ matrices and mean travel times. It is also sufficient to estimate the travel time variance of traffic and detect abnormal mobility conditions, such as traffic jams due to accidents.

\subsection{Stationary Sensing Proof-of-concept}

A proof-of-concept experiment was performed using a stationary setup with sensors on five locations of the urban area of the city of Porto, Portugal. The experiment took place in key entry points to the city depicted in Figure 3. The goal was to measure the average travel times between these 5 points. The experiment took place between 8 and 10 AM, during mornings rush hours.

In Table 1, we show information related to the number of devices detected in this experiment. Table 2 presents a partial O/D matrix, while Table 3 shows average travel times and speed between two given points. 


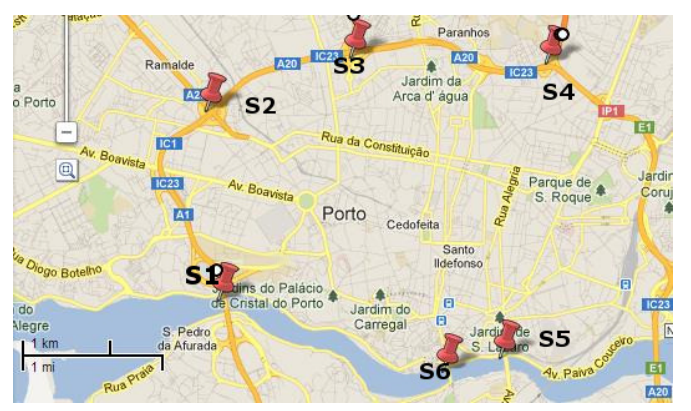

Fig. 3. Monitoring points

Table 1. Information related to discovered devices

Station Discovered Devices Devices/min

\begin{tabular}{lcc}
\hline \hline S1 & 2367 & 29.4 \\
S2 & 2367 & 42.8 \\
S3 & 929 & 8.36 \\
S4 & 4379 & 40.6 \\
S5 & 1016 & 8.40 \\
S6 & 1016 & 6.57
\end{tabular}

Table 2. Example of O/D pairs between stations

From S1 to Devices \% of Devices

\begin{tabular}{lcc}
\hline \hline S2 & 434 & 18.3 \\
S3 & 9 & 0.38 \\
S4 & 80 & 3.38 \\
S5 & 87 & 3.68 \\
S6 & 7 & 0.30
\end{tabular}

Table 3. Average travel times and speeds between points S1, S2

S1 to S2

\begin{tabular}{lc}
\multicolumn{2}{c}{ S1 to S2 } \\
\hline \hline Average Time & $9.63 \mathrm{~min}$ \\
Average Speed & $18.07 \mathrm{~km} / \mathrm{h}$ \\
Fastest & $75.11 \mathrm{~km} / \mathrm{h}-2.32 \mathrm{~min}$ \\
Slowest & $1.84 \mathrm{~km} / \mathrm{h}-89.7 \mathrm{~min}$ \\
Outliers ( $2^{*}$ Average) & $44($ out of 434$)$ \\
\multicolumn{2}{c}{ Without Outliers } \\
\hline \hline Average Time & $4.93 \mathrm{~min}$ \\
Average Speed & $35.31 \mathrm{~km} / \mathrm{h}$ \\
Fastest & $75.11 \mathrm{~km} / \mathrm{h}-2.32 \mathrm{~min}$ \\
Slowest & $9.47 \mathrm{~km} / \mathrm{h}-18.38 \mathrm{~min}$
\end{tabular}

A different long-term experiment using a single sensor was placed in S1. Using only one sensor restricts the utility of our data in characterizing the traffic network globally. However, it still provides an insight on local traffic. Figure 4 shows device volumes for a single day. Rush hours are clearly visible.

Since the sensor was placed at a key entry point to the city, we were able to measure the amount of time that people stayed in the city before leaving again, or vice-versa. Figure 5 shows time intervals between two captures of devices, for 


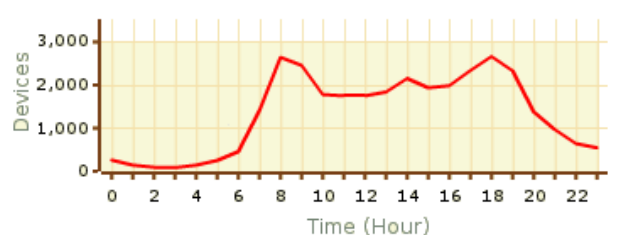

Fig. 4. Device volume for a single day

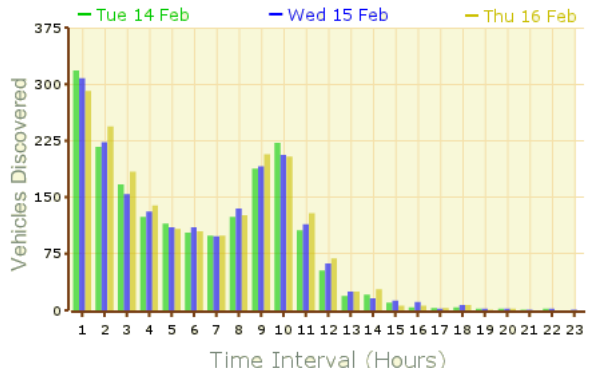

Fig. 5. Time intervals between two detection of a Bluetooth device

three weekdays. The usual 8-hour work schedule is clearly visible. A considerable number of vehicles also stay for short periods of time inside, or outside, the city.

\subsection{Mobile Sensing Proof-of-concept}

Another proof-of-concept experiment was performed for mobile. We opted to use a vehicle with a fixed $30 \mathrm{~km}$ daily commute. The experiment ran for over a month, resulting in 26 hours' worth of Bluetooth discoveries.

Figure 6 shows the evolution of recognized devices over the course of the experiment. We can see a large considerable increase in the percentage of known devices. By the end of the experiment, the probing vehicle already recognized around $30 \%$ of all devices found on the commuting route. As a curiosity, the 36 th commute took an unusual route and the effect is clearly visible.

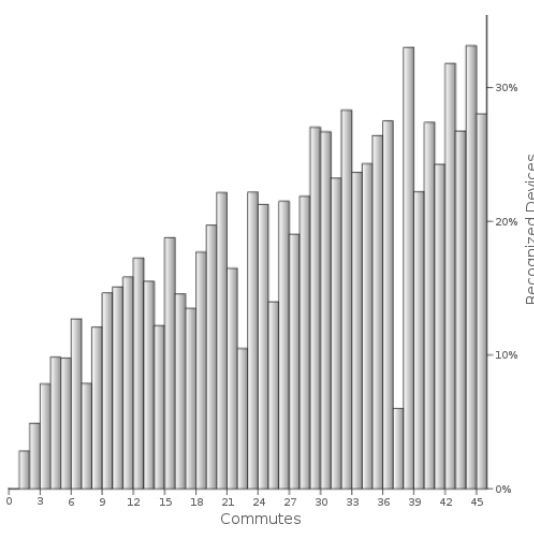

Fig. 6. Percentage of neighbour devices per trip

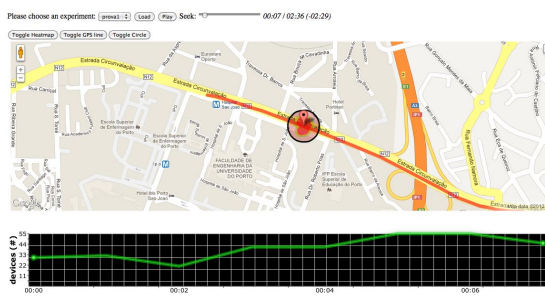

Fig. 7. Mobility activity captured by a mobile Bluetooth scanner 
Depicted in Figure 7 is the web-based visualization tool developed to analyze data captured with mobile sensors. We use a heat-map to depict the number of devices captured. The movement of the probing vehicle may be played as an animation, showing the amount of devices over time and over space.

\section{Conclusion and future work}

Bluetooth sensing technology has been proven to be very promising in mobility studies since it complements with great efficiency traditional traffic detection approaches. Bluetooth data is portable and offers great accuracy in estimating travel times and O-D matrices. In turn, these metrics have various applications such as urban planning or calibration of simulated scenarios both in cases of both traffic and pedestrian monitoring.

In this paper, we presented our take on the traditional approach for Bluetooth monitoring as well as an alternative and novel approach. A mobile sensor provides complementary data to static sensors, and also allows for new studies and new insights. How many people share driving routines? How long does it take for a vehicle to know its neighbors? These questions can be answered by further studying this approach. For example, it would be worthwhile studying further the percentage of recognized vehicles over time and how it changes both with minor and major variations in route or in time of day on which they are taken.

Our early experimental results confirmed that Bluetooth sensing technology could be employed in either setup with promising results. We showed that several varied metrics, from travel times to device recognition rates, could be easily derived from the collected data, with a multitude of applications.

\section{Acknowledgements}

This project has been partially supported by FCT (Fundação para a Ciência e a Tecnologia), the Portuguese Agency for R\&D, under the Bluetooth Sensing Technology project IT/LA/01081/2011 and the grant SFRH/BD/67202/2009.

\section{References}

1. Asmundsdottir, R.: Dynamic od matrix estimation using foating car data. M.sc thesis, Delft University of Technology (2008)

2. Barcelo, J., Montero, L., Marques, L., Carmona, C.: Travel time forecasting and dynamic od estimation in freeways based on bluetooth traffic monitoring. Transportation Research Record: Journal of the Transportation Research Board (Volume 2175), 19-27 (2010)

3. Bullock, D., Haseman, R., Wasson, J., Spitler, R.: Anonymous bluetooth probes for measuring airport security screening passage time: The indianapolis pilot deployment. In: Transportation Research Board 89th Annual Meeting (2010)

4. González, M.C., Hidalgo, C.A., Barabási, A.L.: Understanding individual human mobility patterns. Nature 453(7196), 779-82 (Jun 2008) 
5. Haghani, A., Hamedi, M., Sadabadi, K., Young, S., Tarnoff, P.: Data collection of freeway travel time ground truth with bluetooth sensors. Transportation Research Record: Journal of the Transportation Research Board 2160, 60-68 (2010)

6. Haseman, R., Wasson, J., Bullock, D.: Real-Time Measurement of Travel Time Delay in Work Zones and Evaluation Metrics Using Bluetooth Probe Tracking. Transportation Research Record: Journal of the Transportation Research Board 2169(1), 40-53 (2010)

7. Karlsson, N.: Floating car data deployment \& traffic advisory services. In: Bridging the European ITS Business Cooperation with China (2003)

8. Kostakos, V., Camacho, T., Mantero, C.: Towards proximity-based passenger sensing on public transport buses. Personal and Ubiquitous Computing (Apr 2013)

9. Kostakos, V., ONeill, E.: Cityware: Urban computing to bridge online and realworld social networks. In: Foth, M. (ed.) Handbook of Research on Urban Informatics: The Practice and Promise of the Real-Time City, pp. 195-204. IGI Global (Nov 2008)

10. Kostakos, V., O’Neill, E., Penn, A., Roussos, G., Papadongonas, D.: Brief encounters: sensing, modelling and visualizing urban mobility and copresence networks. ACM Transactions on Computer-Human Interaction 17(1), 1-38 (Mar 2010)

11. Leduc, G.: Road traffic data: Collection methods and applications. In: Working Papers on Energy, Transport and Climate Change. pp. 47-67 (2008)

12. Liebig, T., W.A.: Modelling microscopic pedestrian mobility using bluetooth. In: 4th International Conference on Agents and Artificial Intelligence (ICAART) (2012)

13. Malinovskiy, Y., Wu, Y.J., Wang, Y., Lee, U.K.: Field experiments on bluetoothbased travel time data collection. In: Transportation Research Board 89th Annual Meeting (2010)

14. McPherson, M., Smith-Lovin, L., Cook, J.M.: Birds of a feather: Homophily in social networks. Annual Review of Sociology 27, 415-444 (2001)

15. ONeill, E., Kostakos, V., Kindberg, T., Schiek, A., Penn, A., Fraser, D., Jones, T.: Instrumenting the city: Developing methods for observing and understanding the digital cityscape. In: Dourish, P., Friday, A. (eds.) 8th International Conference of Ubiquitous Computing (UbiComp 2006). Lecture Notes in Computer Science, vol. 4206, pp. 315-332. Springer Berlin Heidelberg, Berlin, Heidelberg (2006)

16. Pels, M., Barhorst, J., Michels, M., Hobo, R., Barendse, J.: Tracking people using bluetooth: Implications of enabling bluetooth discoverable mode. Tech. rep., University of Amsterdam (2005)

17. Sharifi, E., Hamedi, M., Haghani, A., Sadrsadat, H.: Analysis of vehicle detection rate for bluetooth traffic sensors: A case study in maryland and delaware. In: 18th World Congress on on Intelligent Transport Systems (2011)

18. Tarnoff, P.J., Bullock, D.M., Young, S.E., Wasson, J., Ganig, N., Sturdevant, J.R.: Continuing evolution of travel time data information collection and processing. In: Transportation Research Board 88th Annual Meeting (2009)

19. Tsubota, T., Bhaskar, A., Chung, E., Billot, R.: Arterial traffic congestion analysis using bluetooth duration data. In: Tisato, P., Oxlad, L., Taylor, M. (eds.) Australasian Transport Research Forum (Aug 2011)

20. Valerio, D., D'Alconzo, A., Ricciato, F., Wiedermann, W.: Exploiting cellular networks for road traffic estimation: A survey and a research roadmap. In: IEEE 69th Vehicular Technology Conference. pp. 1-5. IEEE (2009)

21. Waadt, A., Wang, S., Bruck, G., Jung, P.: Traffic congestion estimation service exploiting mobile assisted positioning schemes in gsm networks. Procedia Earth and Planetary Science 1(1), 1385-1392 (2009) 
22. Wasson, J.S., Sturdevant, J.R., Bullock, D.M.: Real-time travel time estimates using media access control address matching. ITE Journal 78(6), 20-23 (2008)

23. Young, S.: Bluetooth traffic monitoring technology. Tech. rep., Center for Advanced Transportation Technology, University of Maryland (2008)

24. Young, S.E.: Bluetooth traffic detectors for use as permanently installed travel time instruments. Tech. rep., University of Maryland, College Park; Maryland State Highway Administration (2012) 